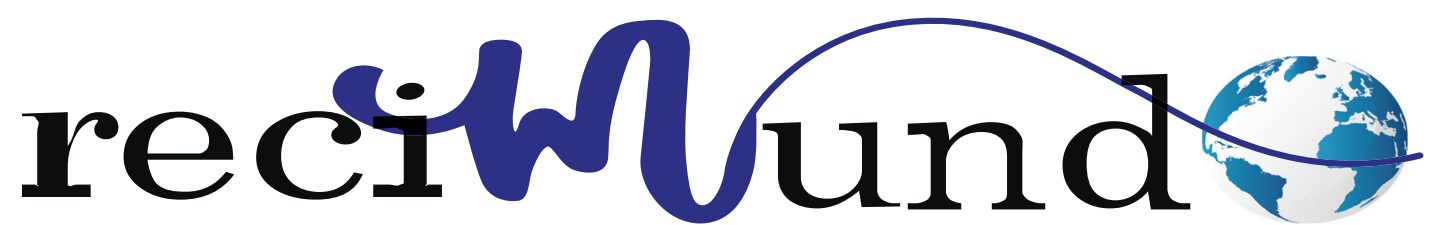

Revista Científica Mundo de la Investigación y el Conocimiento

DOI: 10.26820/recimundo/4.(1).esp.marzo.2020.122-132

URL: http://recimundo.com/index.php/es/article/view/786

EDITORIAL: Saberes del Conocimiento

REVISTA: RECIMUNDO

ISSN: 2588-073X

TIPO DE INVESTIGACIÓN: Artículo de Revisión

CÓdIGO UNESCO: 3201 Ciencias Clínicas

PAGINAS: $122-132$

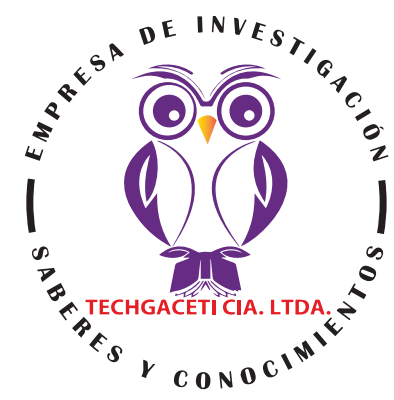

\title{
Manejo de cefalea migrañosa
}

Management of migraine headache

Gestão da enxaqueca

Karla Lisset Chabusa Martínez'; Silvia Eugenia Carbo Baculima²; Jesusov Ronald Guerrero Ramirez³;

Karla Katherine Perez Mendoza ${ }^{4}$

RECIBIDO: 20/11/2019 ACEPTADO: 29/01/2020 PUBLICADO: 05/03/2020

1. Odontóloga; Investigadora Independiente; Guayaquil, Ecuador; lissetchabusa@aol.com; (D) https://orcid. org/0000-0002-3883-0419

2. Médico; Investigadora Independiente; Guayaquil, Ecuador; drascarbo_@hotmail.com; (D) https://orcid. org/0000-0002-5551-4784

3. Licenciada en Enfermería; Investigadora Independiente; Milagro, Ecuador; jesusov2004@hotmail.com; (D) https://orcid.org/0000-0002-8675-4289

4. Licenciada en Enfermería; Investigadora Independiente; Milagro, Ecuador; karla.perez.mendoza.26@gmail. com; (D) https://orcid.org/0000-0002-4115-0297

CORRESPONDENCIA

Karla Lisset Chabusa Martínez

issetchabusa@aol.com

Guayaquil, Ecuador

(C) RECIMUNDO; Editorial Saberes del Conocimiento, 2020 


\title{
RESUMEN
}

El presente trabajo se basa en el estudio de la cefalea migrañosa y como ésta puede afectar significativamente la salud mental y física de la persona que la padece. Se analizaran los tipos de migrañas y su sintomatología y como ésta enfermedad puede perturbar el enfoque social, laboral y monetario en general. El trabajo hace mención a datos estadísticos de la organización mundial de la salud sobre el padecimiento de la enfermedad a escala mundial y el motivo por el cual las mujeres son más propensas que los hombres en padecer estos episodios. En la investigación se observan los pasos a ejecutar para evitar o contrarrestar los dolores, siendo los más comunes; dormir lo suficiente para que el organismo se recupere de la labores diarias, evitar amanecer e ingerir alcohol lo cual es importante debido a que es un fuerte desencadenante de la migraña, llevar una dieta balanceada evitando ciertas comidas que pueda ser causal de detonación, realizar ejercicios que no sean de alto impacto y donde el organismo se sienta cómodo como por ejemplo practicar yoga y realizar ejercicios de relajación que ayuden a concentrarse y eliminar el estrés diario. El objetivo de esta investigación es dar a conocer que llevando una vida sana bajo ciertos parámetros se puede lograr mitigar por completo o en casi su totalidad esta enfermedad que padecen muchos y pocos entienden.

Palabras clave: Cefalea, Migraña, Dolor, Control, Cabeza.

\begin{abstract}
The present work is based on the study of migraine headache and how it can significantly affect the mental and physical health of the person suffering from it. The types of migraines and their symptoms will be analyzed and how this disease can disrupt the social, labor and monetary approach in general. The work mentions statistical data from the world health organization on the disease suffering worldwide and why women are more likely than men to suffer from these episodes. The investigation shows the steps to be taken to avoid or counteract the pain, being the most common; Getting enough sleep so that the body recovers from daily work, avoiding sunrise and drinking alcohol is important because it is a strong trigger for migraine, eating a balanced diet avoiding certain foods that can be the cause of detonation, performing exercises that do not They are of high impact and where the body feels comfortable, for example practicing yoga and performing relaxation exercises that help to concentrate and eliminate daily stress. The objective of this research is to make known that leading a healthy life under certain parameters can mitigate completely or almost completely this disease that many and few suffer from.
\end{abstract}

Keywords: Headache, Migraine, Pain, Control, Head.

\section{RESUMO}

O presente trabalho baseia-se no estudo da enxaqueca e como ela pode afetar significativamente a saúde mental e física da pessoa que sofre dela. Os tipos de enxaqueca e seus sintomas serão analisados e como essa doença pode prejudicar a abordagem social, trabalhista e monetária em geral. O trabalho menciona dados estatísticos da organização mundial de saúde sobre a doença que sofre em todo o mundo e por que as mulheres são mais propensas que os homens a sofrer esses episódios. A investigação mostra as medidas a serem tomadas para evitar ou combater a dor, sendo as mais comuns; Dormir o suficiente para que o corpo se recupere do trabalho diário, evitando o nascer do sol e bebendo álcool é importante porque é um forte gatilho para a enxaqueca, uma dieta equilibrada, evitando certos alimentos que podem ser a causa da detonação, realizando exercícios que não são. de alto impacto e onde o corpo se sente confortável, por exemplo, praticando ioga e realizando exercícios de relaxamento que ajudam a concentrar e eliminar o estresse diário. 0 objetivo desta pesquisa é divulgar que levar uma vida saudável sob certos parâmetros pode mitigar completamente ou quase completamente essa doença da qual muitos e poucos sofrem.

Palavras-chave: Dor de cabeça, enxaqueca, dor, controle, cabeça. 


\section{Introducción}

La mayoría de los humanos han experimentado aquella sensación de dolor de cabeza y muchas veces desconocen la causa que originó tal dolor, imagínense experimentar un dolor intenso en solo una parte de la cabeza, adicional a ello varios síntomas lo acompañan como son las náuseas, intolerancia a la luz y los sonidos, entre otros., en el siguiente artículo se explica a detalle las causas, consecuencias y cómo se puede evitar la cefalea migrañosa para lograr una mejor salud mental y convivencia emocional, visto que esta afecta a gran parte de la población mundial, además de las consecuencias que trae en el área laboral, social, personal.

Según el autor (Beltran J., 2018) "La migraña es una cefalea primaria frecuente e incapacitante, cuyos episodios de dolor suelen durar, al menos, más de cuatro horas si no se tratan. El dolor, de carácter pulsátil, es de intensidad moderada o severa y se localiza habitualmente en un único lado de la cabeza. La migraña es una de las causas más frecuentes de consulta al neurólogo. Estudios epidemiológicos han documentado su alta prevalencia y enorme impacto socioeconómico, siendo, conforme al estudio Global Burden of Disease Survey 2016, el sexto trastorno más prevalente y la segunda causa de discapacidad en el mundo". (p.19).

En entrevista radiofónica de treinta minutos realizada por Marcos Vázquez, autor del blog Fitness Revolucionario, el 17 de febrero de 2016, episodio 55, al Dr. Arturo Goicochea (Neurólogo y Experto en dolor); explica que "la migraña es un dolor real y no justificado, el cual pertenece a un amplio grupo de padecimiento de invalidez como lo es la fibromialgia, indica que es un error de activación de un programa defensivo como lo es el dolor en la cabeza, pero sin que se dé una situación de alarma ni amenaza real en la cabeza". También se explica en la entrevista, que para su manejo se recomienda controlar el miedo el cual es un instinto biológico muy potente, asícomo también entender el dolor y porque se padece, llevar una vida sin estrés y por ultimo una dieta saludable. (Vasquez, 2016).

En entrevista por parte de la CEU al doctor (Goicochea D. A., 2015), explica que:

Las 3 consecuencias de la migraña son la mortificación, la invalidez y la incomprensión. Por otro lado comenta que el cerebro humano no nace aprendido sino que a lo largo del periodo de vida se llena de información y es por eso que deja 3 consejos básicos los cuales son; abrir la mente, tener curiosidad de leer y aprender; por último, practicar lo aprendido con coraje para poder llevar una mejor calidad de vida.

En un artículo publicado por (Bringas, 2010) explica de manera muy técnica y clara lo que significa la enfermedad, y se puede decir que son síntomas bastantes alarmantes para quien lo parece.

La migraña o jaqueca es tal vez el dolor de cabeza más frecuente caracterizado por un desorden crónico neurológico de tipo episódico, recurrente asociado a vómitos, fotofobia, sonofobia, etc. su aparición normalmente se asocian a auras que el paciente percibe previamente. La migraña produce una incapacidad en la persona casi total durante cada episodio, constituyéndose en un problema de salud que requiere un adecuado diagnóstico y tratamiento.

El objetivo de la investigación es aportar evidencias para establecer propuestas y recomendaciones destinadas a mejorar la calidad de vida de las personas con respecto a la enfermedad y tener un poco más de conocimiento sobre la misma.

\section{Metodología}

Esta investigación está enfocada en la Ce- 
falea Migrañosa donde se analizará como la enfermedad perturba de manera significativa la salud mental de las personas que la padecen, el impacto que tiene en su entorno, vida social, personal y por supuesto profesional, para ello se realizará una investigación que brinde orientación acerca de esta enfermedad y cuáles son sus principales causas.

La investigación se ha centrado en textos de medicina neurológica, documentos, artículos científicos disponibles en la web y paginas confiables descartando toda información no confirmada y sin sus respectivas referencias bibliográficas, esto para darle mayor veracidad a la información de este artículo.

\section{Resultados}

Lo primero que se debe exponer en esta investigación es definir la cefalea e identificar la diferencia con respecto a la cefalea migrañosa, la cual afecta a tantas personas a nivel mundial y según lo que indica la Organización Mundial de la Salud (OMS) sobre la enfermedad en página oficial (OMS, 2016) "Las cefaleas (caracterizadas por dolores de cabeza recurrentes) son uno de los trastornos más comunes del sistema nervioso. Son trastornos primarios dolorosos e incapacitantes como la jaqueca o migraña, la cefalea tensional y la cefalea en brotes. También puede ser causada por muchos otros trastornos, de los cuales el consumo excesivo de analgésicos es el más común". Mientras que la Cefalea Migrañosa es considerada por la (OMS, 2016) lo siguiente; Migraña (jaqueca): Es una cefalea primaria. La migraña suele aparecer en la pubertad y afecta principalmente al grupo entre los 35 y los 45 años de edad. Es dos veces más frecuente entre las mujeres que entre los hombres debido a influencias hormonales. Está causada por la activación de un mecanismo encefálico que conduce a la liberación de sustancias inflamatorias y causantes de dolor alrededor de los nervios y vasos sanguíneos de la cabeza. La migraña es recurrente, a menudo dura toda la vida y se caracteriza por episodios consecutivos. Los episodios se caracterizan normalmente por: cefalea (de intensidad moderada a severa), dolor de un solo lado de la cabeza y/o pulsátil, empeora con la actividad física ordinaria, puede durar desde algunas horas hasta 2 o 3 días, náuseas (el síntoma conexo más característico), los episodios se presentan con una frecuencia que varía entre uno al año y uno por semana; en los niños, los episodios suelen durar menos y los síntomas abdominales son más prominentes.

Por lo que se puede demostrar las cefaleas en sus distintos tipos, son los trastornos más comunes del sistema nervioso, y puede llegar a afectar tanto a niños como adultos, estos últimos se estima que la mitad padecen de la enfermedad y estadísticamente las mujeres son más propensas a sufrir de cefaleas debido a los cambios hormonales a los que día a día se enfrentan. Para entender un poco más del trastorno se hace referencia a varias preguntas como las siguientes:

\section{¿Cuán frecuentes son las cefaleas?}

Se calcula que la prevalencia mundial de la cefalea (al menos una vez en el último año) en los adultos es de aproximadamente $50 \%$. Entre la mitad y las tres cuartas partes de los adultos de 18 a 65 años han sufrido una cefalea en el último año, y el 30\% o más de este grupo han padecido migraña. La cefalea que se presenta 15 días o más cada mes afecta de un $1,7 \%$ a un $4 \%$ de la población adulta del mundo. A pesar de las variaciones regionales, las cefaleas son un problema mundial que afecta a personas de todas las edades, razas, niveles de ingresos y zonas geográficas. (OMS, 2016) La (OMS, 2016) precisa que la cefalea no solo es dolorosa sino también incapacitante. De acuerdo al Estudio de la Carga Mundial de Morbilidad, actualizado en 2013, por

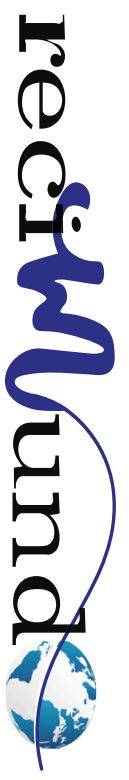


si sola representó la sexta causa mundial de años perdidos por discapacidad. Entonces; ¿Qué carga imponen las cefaleas?

La cefalea no solo es dolorosa sino también incapacitante. En el Estudio de la Carga Mundial de Morbilidad, actualizado en 2013, la migraña por sí sola representa la sexta causa mundial de los años perdidos por discapacidad. Colectivamente, las cefaleas son la tercera causa. Las cefaleas imponen además una carga personal bajo la forma de intenso sufrimiento, menoscabo de la calidad de vida y costos económicos. Las cefaleas frecuentes, así como el temor constante con respecto al siguiente episodio, dañan la vida familiar, las relaciones sociales y el trabajo. Los esfuerzos prolongados que supone habérselas con una cefalea crónica pueden asimismo predisponer a la aparición de otras enfermedades; por ejemplo, la depresión y la ansiedad son significativamente más frecuentes en las personas aquejadas de migraña que en las sanas.

La (OMS, 2016) en su artículo sobre cefaleas comenta que la carga económica y social de las cefaleas son bastantes preocupantes para la salud pública, debido en principio la discapacidad de las personas y segundos los costos económicos que acarrea para la sociedad que la padece.

Se calcula que los costos económicos para la sociedad, relacionados principalmente con las horas de trabajo que se pierden y la mengua de la productividad son enormes. En el Reino Unido, por ejemplo, cada año se pierden unos 25 millones de días de trabajo o de escuela por causa de la migraña; este costo económico puede ser igual al que causan la cefalea tensional y la cefalea diaria crónica combinada. La cefalea es una de las causas principales de consulta médica; en un estudio, una tercera parte de las consultas neurológicas fueron motivadas por este síntoma.
A continuación se muestra una tabla diseñada por el autor (Bazan, 2015), y explica que las migrañas se clasifican en 2 tipos (con aura y sin aura), es importante, diferenciarlas y de esta manera poder tomar las medidas que más se adapten al momento de presentar alguna crisis migrañosa.

A. El diagnóstico de migraña con aura requiere cuando menos dos crisis de cefalea, precedidos de los fenómenos señalados en B, C y D

B. Aura consistente en por lo menos uno de los siguientes (no debilidad muscular).

1. Síntomas Visuales, completamente reversibles, uno de características positivas como luces titilantes, manchas luminosas o líneas luminosas $u$ otras negativas como pérdida de visión.

2. Síntomas somato sensoriales completamente reversibles, uno con características positivas como hincadas o pinchazos y otros negativos como adormecimiento.

3. Disfasia transitoria, totalmente reversible.

C. Al menos dos de los siguientes

1. Síntomas visuales homónimos y/o síntomas sensoriales unilaterales.

2. Al menos un episodio de aura que evoluciona en casi 5 minutos y/o diferentes en sucesión cada 5 minutos.

3. Cada aura dura más de 5 minutos, pero menos de 1 hora.

D. Cefalea que cumple los criterios B-D de migraña sin aura, el dolor comienza durante el aura o aparece post aura, en un tiempo no mayor de 60 minutos.

E. La cefalea no puede ser atribuida a otra afección.

Tabla 1. Migraña con Aura o migraña clásica

Fuente: (Bazan, 2015) 
A. Cuando menos 5 ataques de cefalea que cumplen lo señalado desde B a D.

B. Cada ataque dura entre 4 a 72 horas (con terapia ineficaz o ausente).

C. Dolor con al menos dos de las siguientes características:

1. Localización unilateral.

2. Cualidad pulsátil.

3. Dolor de intensidad moderada a severa.

4. Causado o agravado por la actividad física rutinaria (caminar o subir escaleras, etc.).

D. Presencia durante la cefalea a alguno de los siguientes síntomas:

1. Náusea y/o vómito.

2. Fotofobia y sonofobia.

E. La cefalea no es atribuible a otra enfermedad.

Tabla 2. Migraña sin aura o migraña común

Fuente: (Bazan, 2015)

En el ámbito clínico, la barrera principal es el desconocimiento del personal sanitario. Por término medio, en todo el mundo se dedican únicamente cuatro horas a la instrucción sobre las cefaleas en los planes de estudio de licenciatura. El diagnóstico y tratamiento profesional se establece en una minoría de estos pacientes: un $40 \%$ de los aquejados de migraña y cefalea tensional, pero apenas un $10 \%$ de los que sufren la cefalea por uso excesivo de analgésicos. (Pujol, 2018)

La gente tampoco sabe mucho de las cefaleas. La población general no considera que las cefaleas sean una enfermedad grave porque se presentan generalmente por episodios, no son mortales ni se contagian. Las tasas bajas de consulta en los países desarrollados pueden indicar que muchos enfermos desconocen la existencia de tratamiento eficaz; se calcula que la mitad de las personas que padecen cefalea se tratan ellas mismas. Como muchos gobiernos pretenden limitar los costos de la asistencia sanitaria, no reconocen que las cefaleas imponen una carga considerable a la sociedad. Es probable que no se den cuenta de que los costos directos del tratamiento de las cefaleas son ínfimos si se comparan con los enormes ahorros que podrían lograrse en el apartado de los costos indirectos (por ejemplo, al reducir los días de trabajo perdidos) si se asignaran recursos suficientes a su tratamiento.

Normalmente siempre hay un desencadenante para cualquier enfermedad y la cefalea migrañosa no es la excepción, existen ciertos detonantes que contribuyen, pero si se logra manejar pueden llevar una vida normal y así evitar un episodio, como las personas que no padecen de esta enfermedad, así como lo destaca el artículo publicado el 07 de Marzo de 2018, (Pujol, 2018). A continuación las posibles causales de activación;

Relacionados con la comida: existen algunos alimentos que pueden influir en la aparición de la migraña como son: queso, comida china, chocolate, cítricos, productos lácteos, pescado ahumado, embutidos con contenido de nitratos (salami, hotdog, tocino), frutos secos, arenques, vinagre, tomates y trigo, además del consumo de alcohol. Por otro lado, el ayuno prolongado puede presentar el mismo efecto.

Causas hormonales: el hecho de que la migraña sea mucho más frecuente entre la población femenina (hasta el doble), tiene que ver con los cambios hormonales en relación con el ciclo menstrual y la menopausia, que pueden también provocar ataques. De la misma manera, los anticonceptivos hormonales pueden afectar a estas cefaleas.

Factores físicos: realizar actividades intensas como el levantamiento de pesas, los esfuerzos importantes y, en ocasiones, hasta el simple hecho de flexionarse o agacharse pueden ser desencadenantes. 
Problemas emocionales: el estrés, la depresión, el insomnio, estados de shock pueden igualmente convertirse en precipitantes de la migraña.

Factores ambientales: a menudo la exposición a una luz muy brillante, el sol intenso o los cambios muy bruscos de temperatura o de presión atmosférica pueden afectar considerablemente, al igual que los sonidos agudos y elevados, los olores penetrantes o los baños con agua muy caliente.
Pueden existir otros factores que colaboren con la activación de la cefalea migrañosa en las personas que la padecen; sin embargo los más comunes son los que se mencionan en el artículo de la página web tu canal salud y cuya explicación está bastante clara. Este tipo de patologías se puede mitigar o evitar siguiendo las recomendaciones que a su juicio les parezca más conveniente y según la forma de vida que se lleve. Nunca es tarde para mejorar su salud y más cuando existen métodos, guías, asesorías, talleres para lograr extenuar todas las complicaciones que les aquejan.

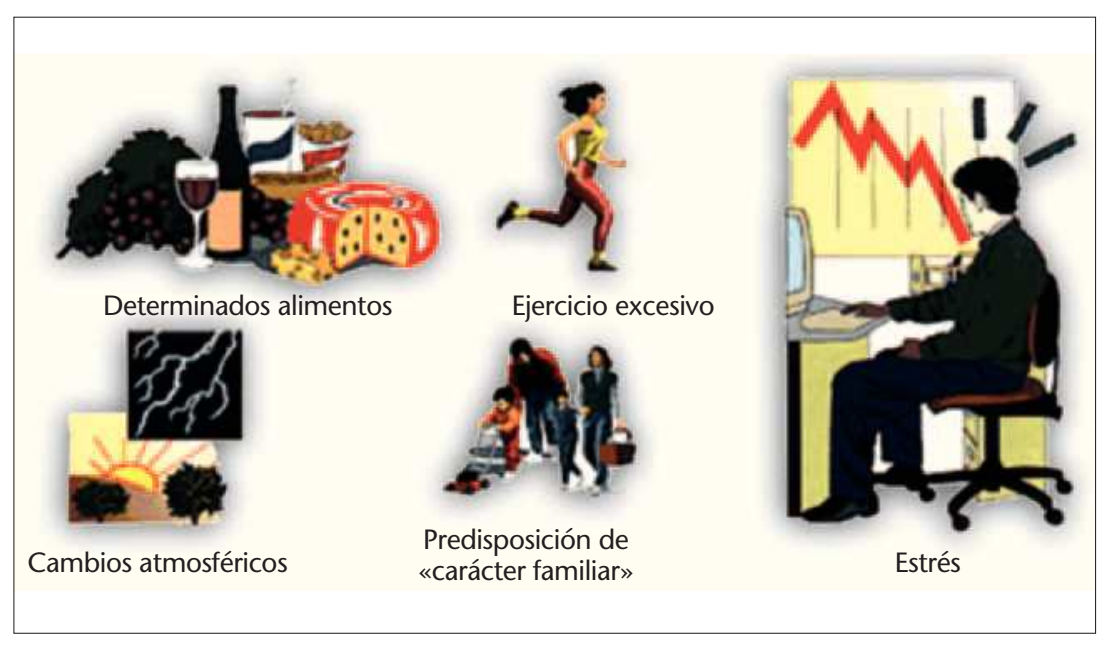

Figura 1. Factores desencadenantes de migraña

Fuente: (Gomez, 2006)

En la imagen se puede destacar ciertos desencadenantes de la migraña; sin embargo existen múltiples factores como los que se mencionaron más arriba y como lo menciona tu canal de salud.

Para los dolores de cefalea migrañosa existen múltiples tratamientos farmacéuticos los cuales dependiendo del tipo de cefalea, el neurólogo recetara para poder calmar y mitigar todos esos síntomas adicionales que acompañan la tan complicada enfermedad, así como los explica (Takeuchi, 2008) en su artículo "De acuerdo a un esquema estratificado, los principios del tratamiento del ataque agudo de la migraña se basan en clasificar las características según; severidad de los ataques, tiempo o latencia para alcanzar la intensidad máxima (tiempo de pico) y presencia de síntomas asociados". Se puede decir que se tiene que evaluar primero el tipo de migraña junto con sus síntomas para poder dar el mejor tratamiento posible, así mismo la investigación de la actualización de la migraña expresa que dependiente de la severidad del ataque se puede tomar algún analgésico tales como: 


\begin{tabular}{|c|c|c|c|}
\hline Leves & Moderados & Severos & $\begin{array}{c}\text { Extremadamente } \\
\text { severos }\end{array}$ \\
\hline $\begin{array}{l}\text { - aspirina (750 } \\
\text { mg-1 g). } \\
\text { - acetaminofén (1 } \\
\text { g) } \\
\text { - otros AlNES } \\
\left(500 \mathrm{mg}^{-1} \mathrm{~g}\right)\end{array}$ & $\begin{array}{l}\text {-Aspirina. } \\
\text {-AINES (naproxén } \\
\text { sódico, } 750 \text { mg). } \\
\text { - Ergotamina (1 } \\
\text { mg). } \\
\text { - Isometepteno } \\
\text { (una tableta o } 40 \\
\text { gotas). } \\
\text { - acetaminofén } \\
\text { más codeína (una } \\
\text { tableta) o alguno } \\
\text { de los triptanes: } \\
\text { - sumatriptán } \\
\text { (50mg). } \\
\text { - zolmitriptán (2,5 } \\
\text { mg). } \\
\text { - naratriptán. } \\
\text { - rizatriptán. } \\
\text { - almotriptán. } \\
\text { - eletriptán. }\end{array}$ & $\begin{array}{l}\text { - acetaminofén } \\
\text { más codeína (una } \\
\text { tableta). } \\
\text { - alguno de los } \\
\text { triptanes (una } \\
\text { tableta). }\end{array}$ & $\begin{array}{l}\text { - haloperidol 0,1 } \\
\text { mg/K/IV, } \\
\text { - AINE por vía } \\
\text { endovenosa. } \\
\text { - dexametasona (4 } \\
\text { mg IV cada } 8 \text { h por } \\
\text { un periodo de } 72 \\
\text { h). }\end{array}$ \\
\hline
\end{tabular}

Tabla 3. Tipos de ataques

Fuente: (Takeuchi, 2008)

El autor (Takeuchi, 2008), define los ataques migrañosos de acuerdo a lo siguiente:

- Ataques leves se definen como aquellos que no interfieren con la actividad diaria y que en su mayoría pueden autolimitarse o ceder con analgésicos simples.

- Los ataques moderados son descritos por los pacientes como un dolor de intensidad entre 5 y 7 en una escala de 10, y aunque pueden interferir con la actividad, el paciente no tiene que interrumpirla; en su mayoría pueden ser controlados.

- Los ataques severos, el paciente tiene que interrumpir sus actividades.
- Para los ataques extremadamente severos, es decir, aquellos que incapacitan al paciente por tiempo prolongado y no responden a las medidas descritas, y el status migrañoso, es preciso recurrir a la hospitalización. Los opiáceos sólo se utilizan cuando no hay respuesta a todas las acciones anteriores, considerando el riesgo de adicción. 


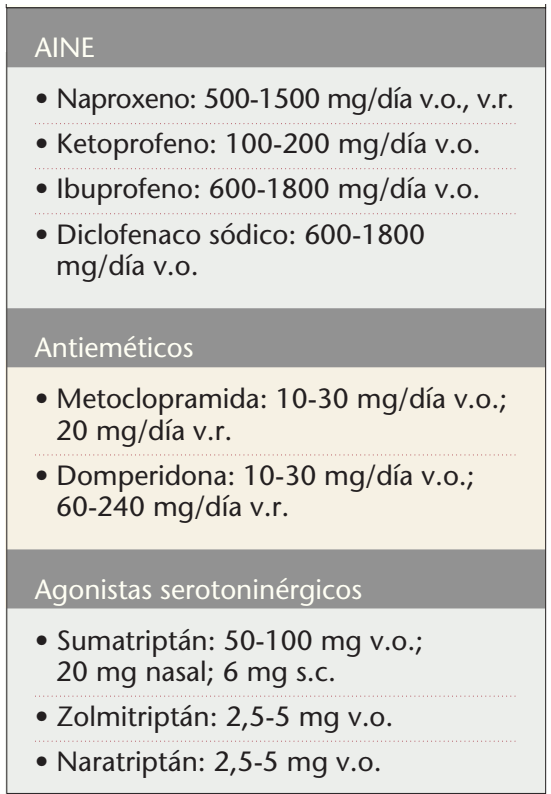

Tabla 4. Tratamiento Sintomático

Fuente: (Gomez, 2006)

Es importante mencionar que el Dr. (Goicochea A. , 2016), viene trabajando el dolor de una manera muy peculiar y es mediante el control del dolor a través de ciertas técnicas y talleres basados en saber controlar el miedo y saber entenderlo, a partir de allí se comienza a educar el cerebro, solo se necesita abrir la mente de manera metafórica, tener la curiosidad de leer todos los libros y el material que el doctor suministra y aplicarlo en el día a día con mucho coraje y entusiasmo.

En la actualidad se implementa un tratamiento bastante interesante que consiste en explicar a los pacientes cómo funciona el dolor. Según parece el cerebro va asimilando esas instrucciones y, gracias a la neuroplasticidad, se reestructura poniendo fin al dolor. Los resultados son mucho mejores, muy por encima del modelo médico tradicional; así lo expresa la psicóloga. (Pascual, 2020).

Pascual recuerda que lo que podría mantener y empeorar está patología son todos los intentos que la persona pone en marcha para combatirlos. En la migraña, como en las fobias, las principales soluciones inten- tadas que no funcionan son la evitación, las precauciones. Por ejemplo, los pacientes viven con gran desasosiego con la posible aparición de un nuevo brote migrañoso, lo que genera una mayor ansiedad que con frecuencia acaba en la profecía autocumplida. La terapia breve estratégica dictada por la psicóloga (Pascual, 2020), consiste en lo que se menciona a continuación:

Es conocida por dar prescripciones extrañas e ilógicas que solventan el problema. ¿Por qué son ilógicas? Porque hemos observado que la mayoría de los problemas psicológicos tienen una base totalmente ilógica y por lo tanto su solución debe de adaptarse a esa base ilógica. El cambio lo buscamos o bien a través de un uso de comunicación persuasiva capaz de llegar a tocar el corazón y cambiar la visión del paciente y llevando a la persona a la acción: hacer algo nuevo que le produzca una experiencia emocional correctiva que haga percibir su realidad problemática con otros ojos.

Se puede observar ciertos consejos para las personas que por primera vez están padeciendo la enfermedad. (AEMICE, 2020) 
-Para descartar otras enfermedades, puede realizarte un examen físico general y un examen neurológico.

-Para el diagnóstico de la migraña con aura visual o retiniana puede ser necesaria una evaluación oftalmológica que permita descartar posibles enfermedades del ojo.

-Para el diagnóstico de la migraña menstrual se recomienda completar un calendario del registro del dolor, durante 3 ciclos menstruales consecutivos. Deberás anotar los días de inicio y fin de la regla y los días con dolor en ese periodo.

Pueden ser necesarias pruebas diagnósticas complementarias, en función de tu historial clínico.

\section{Conclusiones}

Luego de la investigación realizada sobre la cefalea migrañosa se obtuvo que la enfermedad la padecen la mitad de los adultos en el mundo y la mayoría mujeres debido a los cambios hormonales, los cuales llevan al desencadenamiento de la migraña, para mitigarlo es necesario seguir ciertos parámetros que ayuden a mejorar la calidad de vida, donde algunos de ellos son: llevar una vida sin tanto estrés, una buena y balanceada alimentación, evitar bebidas oscuras y chocolates, tratar de no excederse con los ejercicios de alto impacto, realizar ejercicios de relación como el yoga, adicional a ellos es importante meditar en lugares donde la iluminación y el sonido no afecten el entorno donde se realiza dicha actividad.

En el desarrollo de la investigación también se observó que existen ciertos fármacos que son recetados por el especialista de acuerdo al nivel e intensidad de dolor que se padece en el momento, que han sido mencionados en el trabajo de investigación. Para tratar la cefalea migrañosa es de suma importante que los involucrados entiendan que necesitan de profesionales sanitarios que estén bien capacitados para que la detección y el diagnóstico del trastorno sea lo más exacto posible y puedan proporcionar el tratamiento adecuado de manera eficaz, esto con el fin de poder lograr una mejor calidad de vida.

En definitiva, la persona con migraña tiene una evidente susceptibilidad genética en muchos casos de padecer los peculiares episodios dolorosos, en respuesta a determinadas circunstancias externas, conocidas en conjunto como factores precipitantes o "disparadores".

El estrés, de cualquier causa, según lo investigado, es una de las principales causas de migraña, es el disparador más frecuente del episodio doloroso en los pacientes. La experiencia clínica muestra asimismo, que tanto el estrés como los otros desencadenantes, tienen diferente umbral de activación en una misma persona.

Finalmente se conoce que muchas personas diagnosticadas de migraña, logran un buen control de sus molestias, simplemente, identificando y luego evitando sus personales factores de riesgo.

\section{Bibliografía}

AEMICE. (28 de 02 de 2020). Asociacion española de migraña y cefalea. Recuperado el 28 de 02 de 2020, de https://www.dolordecabeza.net/dolor-de-cabeza/migrana/

Bazan, D. C. (2015). Recuperado de: https://es.slideshare.net/cristiancarpio777/manejo-de-la-cefalea.

Beltran J., L. A. (2018). Impacto y situación de la migraña en España atlas 2018. Sevilla: Universidad de Sevilla año 2018.

Bringas, L. D. (2010). La Migraña. Scielo, http:// www.scielo.org.pe/pdf/amp/v27n2/a09v27n2. pdf.

Goicochea, D. A. (09 de 12 de 2015). Seminario migrañas cuestion de cerebro. https://www.youtube. com/watch?v=yPG6WUOx800. (C. e. 3, Entrevistador) Vitoria, España.

Gomez, A. E. (03 de 2006). Cefalea cronica diaria. Elsevier, https://www.elsevier.es/es-re-

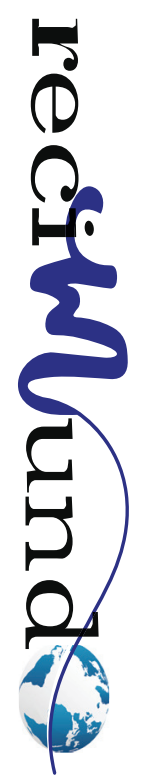


vista-offarm-4-articulo-cefalea-cronica-diaria-13085788.

Migrañas y el dolorEpisodio 55https://www.fitnessrevolucionario.com/2016/02/17/episodio-55-arturo-goicochea-sobre-migranas-y-dolor/

OMS. (08 de 04 de 2016). Recuperado el 28 de 02 de 2020, de Recuperado de: https://www.who.int/ es/news-room/fact-sheets/detail/headache-disorders

Pascual, J. (28 de 02 de 2020). Terapia Breve Estratégica de Barcelona. (D. Sojo, Editor) Recuperado el 28 de 02 de 2020, de http://www.juliapascual. com/tratamiento-migrana/
Pujol, A. (07 de 03 de 2018). https://www.tucanaldesalud.es/es/tusaludaldia/articulos/dolor-cabeza-cefalea-migranosa. Obtenido de Tu canal de salud. es quiron salud.

Takeuchi, Y. (09 de 2008). https://www.acnweb.org/ acta/2008_24_S3_44.pdf.

Vasquez, M. (17 de 02 de 2016). Migraña y el doIor [Audio en podcast]. Recuperado de: https:// www.fitnessrevolucionario.com/2016/02/17/episodio-55-arturo-goicochea-sobre-migranas-y-dolor/. (A. Goicochea, Entrevistador) Vitoria, España.

\section{CITAR ESTE ARTICULO:}

Chabusa Martínez, K., Carbo Baculima, S., Guerrero Ramirez, J., \& Perez Mendoza, K. (2020). Manejo de cefalea migrañosa. RECIMUNDO, 4(1(Esp)), 122132. doi:10.26820/recimundo/4.(1).esp.marzo.2020.122-132 